Www.jmscr.igmpublication.org

Index Copernicus Value: 79.54

ISSN (e)-2347-176x ISSN (p) 2455-0450

crossrefDOI: https://dx.doi.org/10.18535/jmscr/v7i2.45

\title{
Computed Tomographic Characterisation of Large Abdominal Mass - A Hospital Based Descriptive Study
}

\section{Dr Muthiah.P ${ }^{1}$, Dr Pradeep Vel M..$^{{ }^{*}}$, Dr Adaikappan. M. ${ }^{3}$}

${ }^{1}$ Associate Professor, Department of Radio Diagnosis, Rajah Muthiah Medical College, Annamalai Nagar,

Chidambaram, Tamilnadu, India

${ }^{2}$ Resident, Department of Radio Diagnosis, Rajah Muthiah Medical College, Annamalai Nagar,

Chidambaram, Tamilnadu, India

${ }^{3}$ Professor and Head, Department of Radio Diagnosis, Rajah Muthiah Medical College, Annamalai Nagar, Chidambaram, Tamilnadu, India

*Corresponding Author

Dr Pradeep Vel M

Resident, Department of Radio Diagnosis, Rajah Muthiah Medical College, Annamalai Nagar,

Chidambaram, Tamilnadu, India.608002

Email: pradeepmvel@gmail.com

\begin{abstract}
Introduction: Patients presenting with a large abdominal mass are to be investigated judiciously and accurate diagnosis is always needed for surgical or medical treatment planning. With newer technological innovations and thinner slice fast imaging by MSCT/MDCT, the confidence level of the radiologist is further improved in diagnosis.
\end{abstract}

Aim: To assess the efficacy of the Computed Tomography in assessing the lesion and to analyze its ability to characterize the lesions by imaging and predict its benign or malignant nature.

Materials and Methods: Prospective study of 410 patients subjected to CT with a clinical diagnosis of large intra abdominal mass was done. The study was carried over a period of 18 months at our institute. All data were recorded and analysed.

Results: Out of 26 cases included in our study after applying exclusion criteria, it is observed female [70\%] preponderance over men [30\%] and ovarian mass lesions 46\% and peritoneal/Mesenteric mass lesions [23\%] were the leading causes. uterine [7.6\%], Gastro intestinal[7.6\%], Hepatic [3.8\%], Pancreatic [3.8\%] and Gall bladder lesions form the rest of our study. CT characteristic features of all encountered lesions were comparable with various previous studies.

Conclusion: Though, Conventional ultrasonography is the primary screening tool for patients with a large abdominal mass, for accurate and confident diagnosis, the role of CT in further characterizing the lesion is needed for the clinician for further treatment and it also serves a roadmap if surgery is contemplated.

Keywords: Computed Tomography, Large abdominal mass, Characterization.

\section{Introduction}

Technological innovations and advancements in Computed Tomography (CT), Magnetic
Resonance Imaging (MRI) and Ultrasonography have immensely helped us improve our ability and guide us to confidently diagnose abdomino- 
pelvic neoplasms and for further follow up in monitoring treatment efficacy.

Plain radiographs and positive and negative contrast techniques are the basic fundamental imaging modalities. In the recent times, they have largely been challenged by the supremacy of the cross-sectional imaging. Ultrasonography, MRI along with Positron Emission Tomography (PET) are new ways and their diagnostic role is increasing. The suspected pathology, the clinical findings and the probable organ involved determines the modality to be chosen.

The characteristic appearances of primary and secondary abdominal lesions and a rational approach to the differential diagnosis of them depicted by Computed Tomography done for patients presenting to our department with clinically palpable masses were presented in this article.

\section{Materials and Methods}

A total of 410 patients were subjected to CT study with clinical suspicion of large intra-abdominal mass or indeterminate ultrasonographic features. The study was carried out between January 2016 and August 2017 over a period of 18 months at our institute. Our inclusion criteria were any patient with 18 years of age and above and those having an intra abdominal mass with size greater than $15 \mathrm{~cm}$ in its largest dimension. Distended bowels of any cause, distended urinary bladder and benign solid organ enlargement were our exclusion criteria. After applying exclusion criteria, about 26 cases were included in our study and the images were interpreted and analysed.

\section{Statistical Analysis}

Statistical analysis was done by applying descriptive statistics. The categorical data is expressed in the manner of percentage and presented by tables.

\section{Results}

Out of 26 patients included in our study, we had a female preponderance of $70 \%$ and $65 \%$ patients were in the mean age group between 35 and 55 . [Chart -1 and 2]. The size and calculated volumes of all the mass lesions in all three dimensions were measured and plotted in the chart. [Chart - 3 and 4]. We have encountered ovarian mass lesions [46\%], peritoneal/Mesenteric lesions [23], uterine [7.6\%], gastro intestinal [7.6\%], hepatic [3.8\%], pancreatic [3.8\%] and gall bladder [3.8\%] lesions in our study. [Chart 5]. Developmental causes accounted for $19 \%$, Acquired lesions accounted for $15 \%$ and neoplastic etiology $66 \%$ with benign and malignant cases representing $47 \%$ and $53 \%$ respectively. [Chart 6 and 7]. $62 \%$ cases were cystic, $23 \%$ were solid and $15 \%$ constitute mixed lesions. [Chart 8]. The final pathological diagnosis of all analysed cases were given in Chart 9.

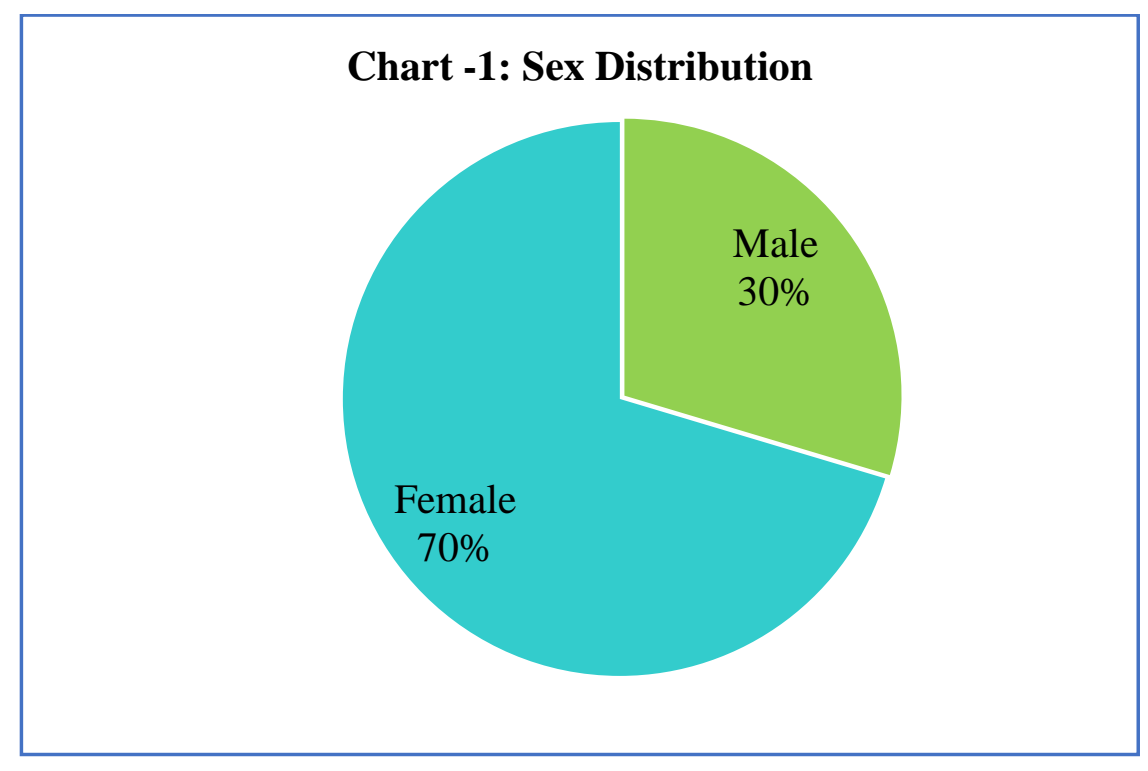



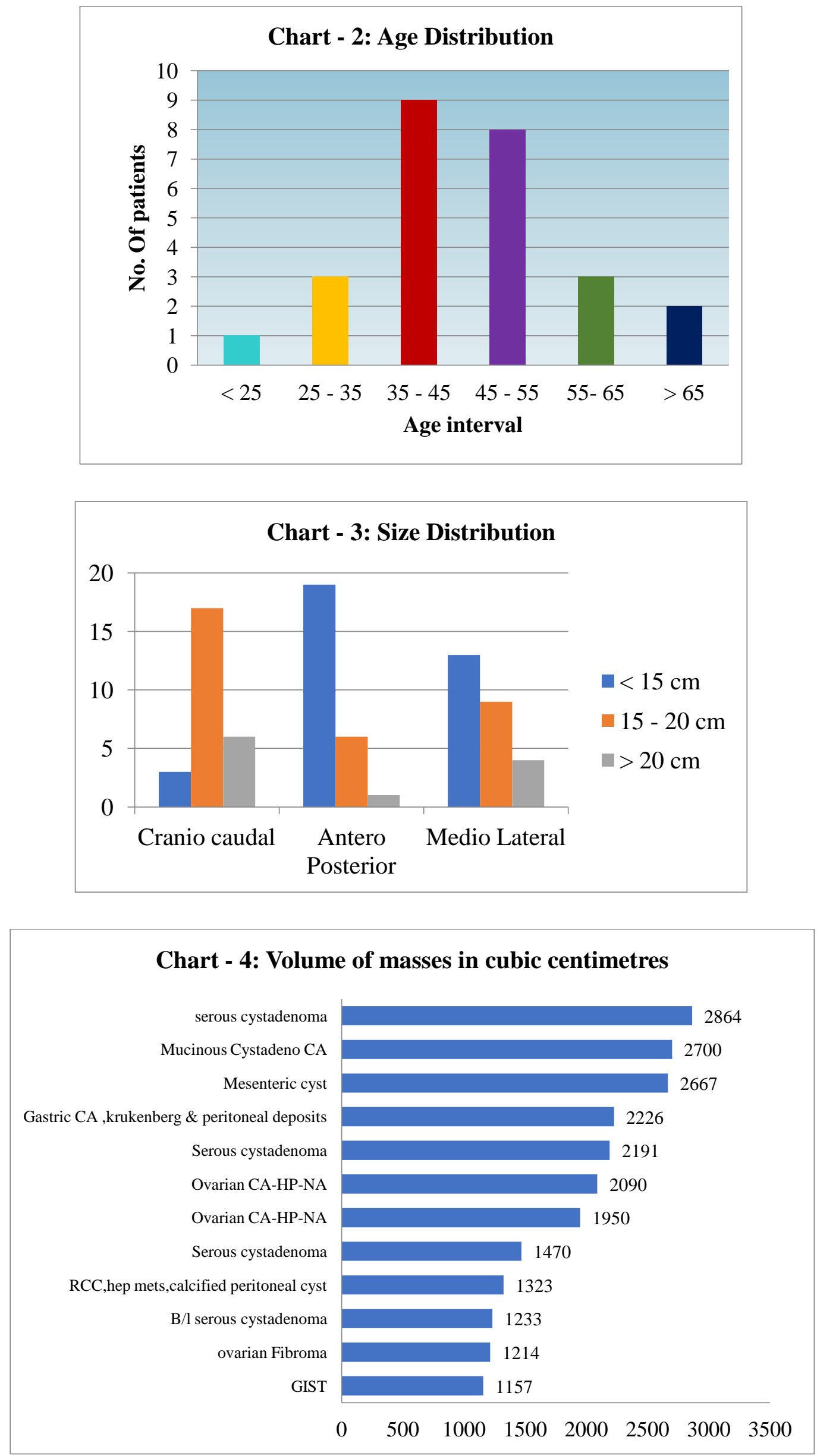

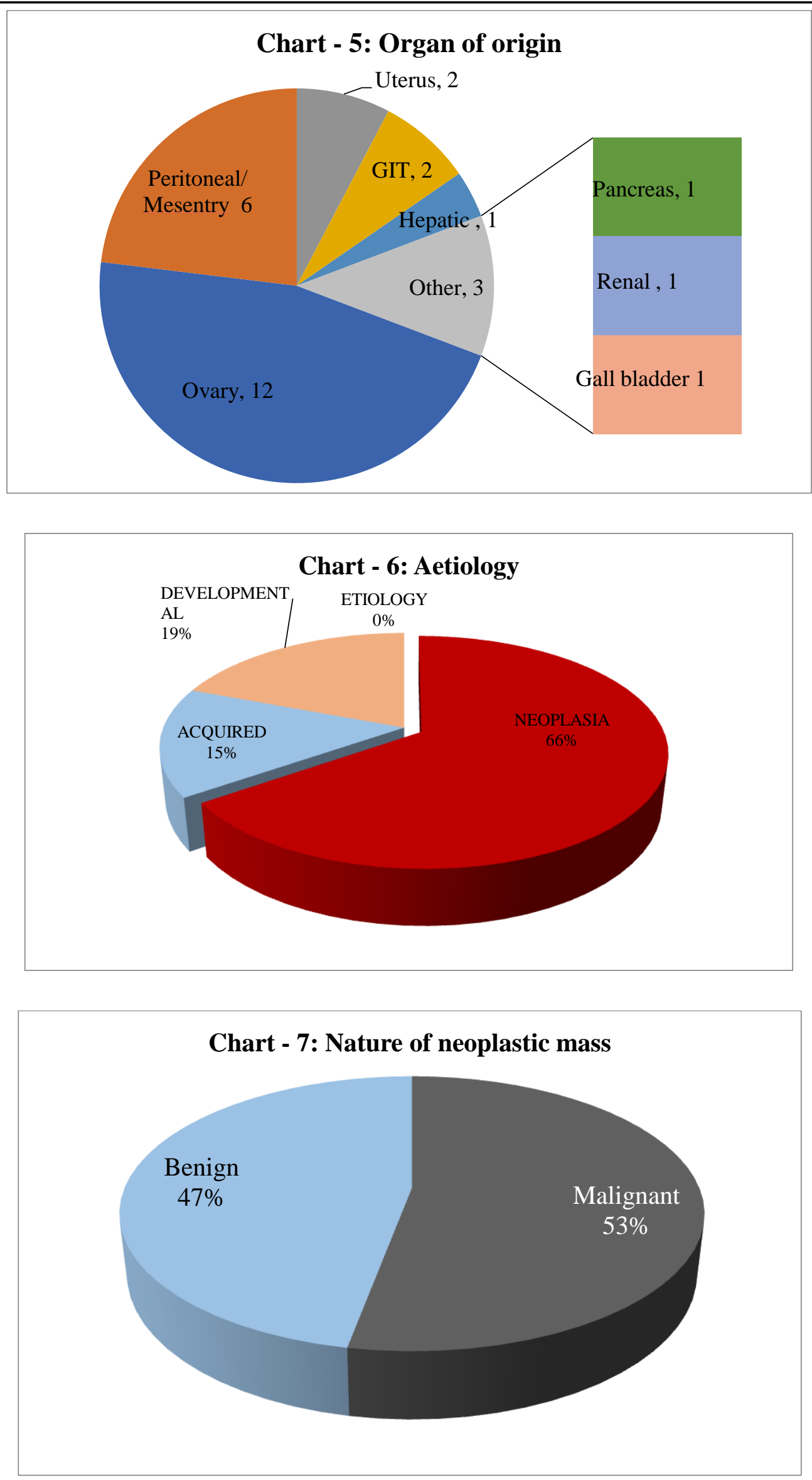


\section{Chart - 8: Gross morphology}

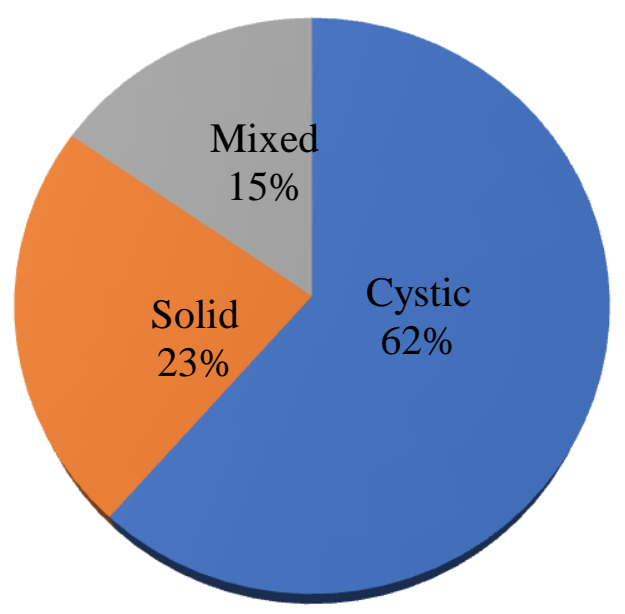

\section{Chart - 9: Pathology}

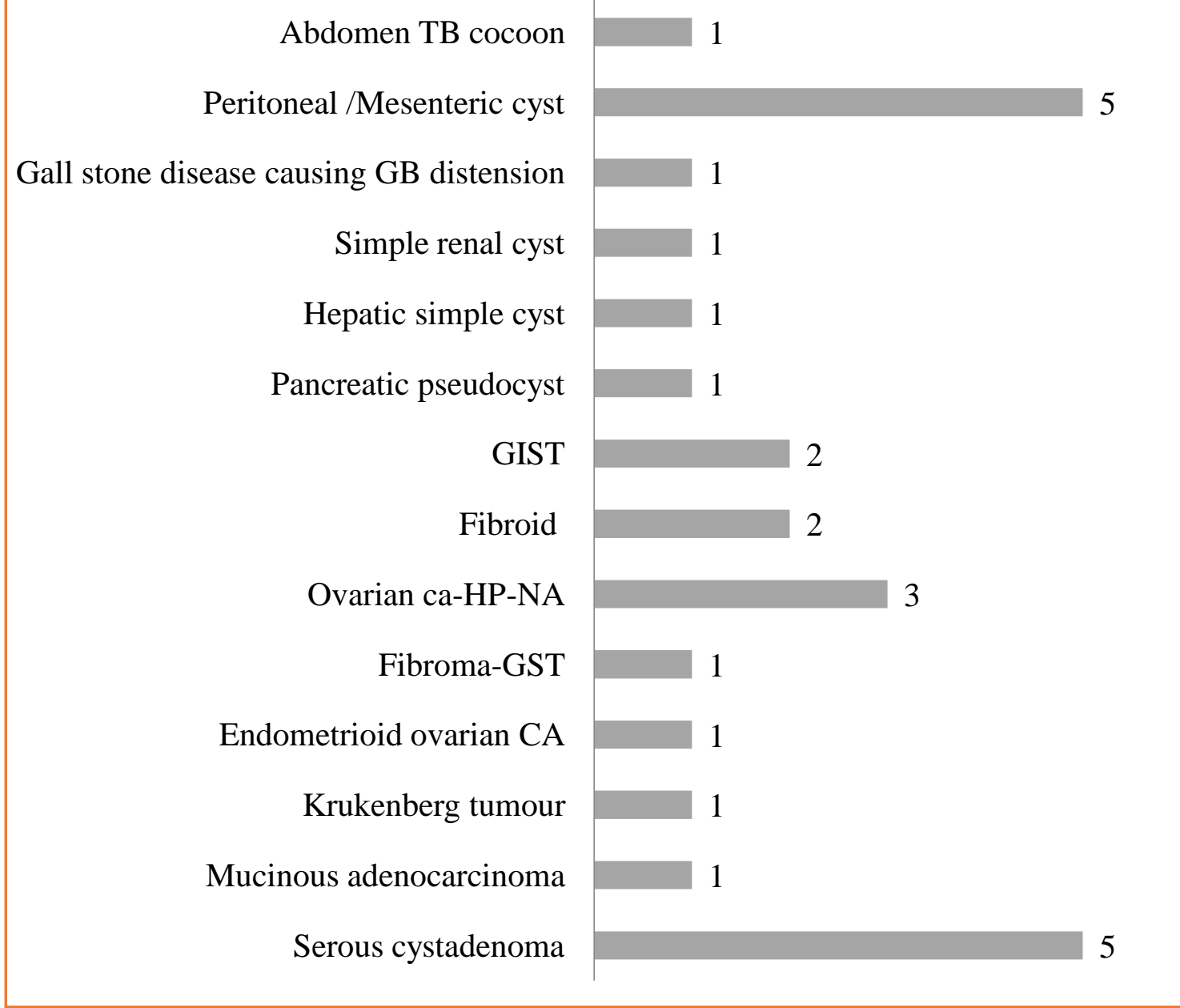




\begin{tabular}{|l|l|}
\hline JMSCR Vol |07||Issue ||02||Page 235-243||February & 2019 \\
\hline
\end{tabular}

Fig-1: Mucinous cystadenocarcinoma
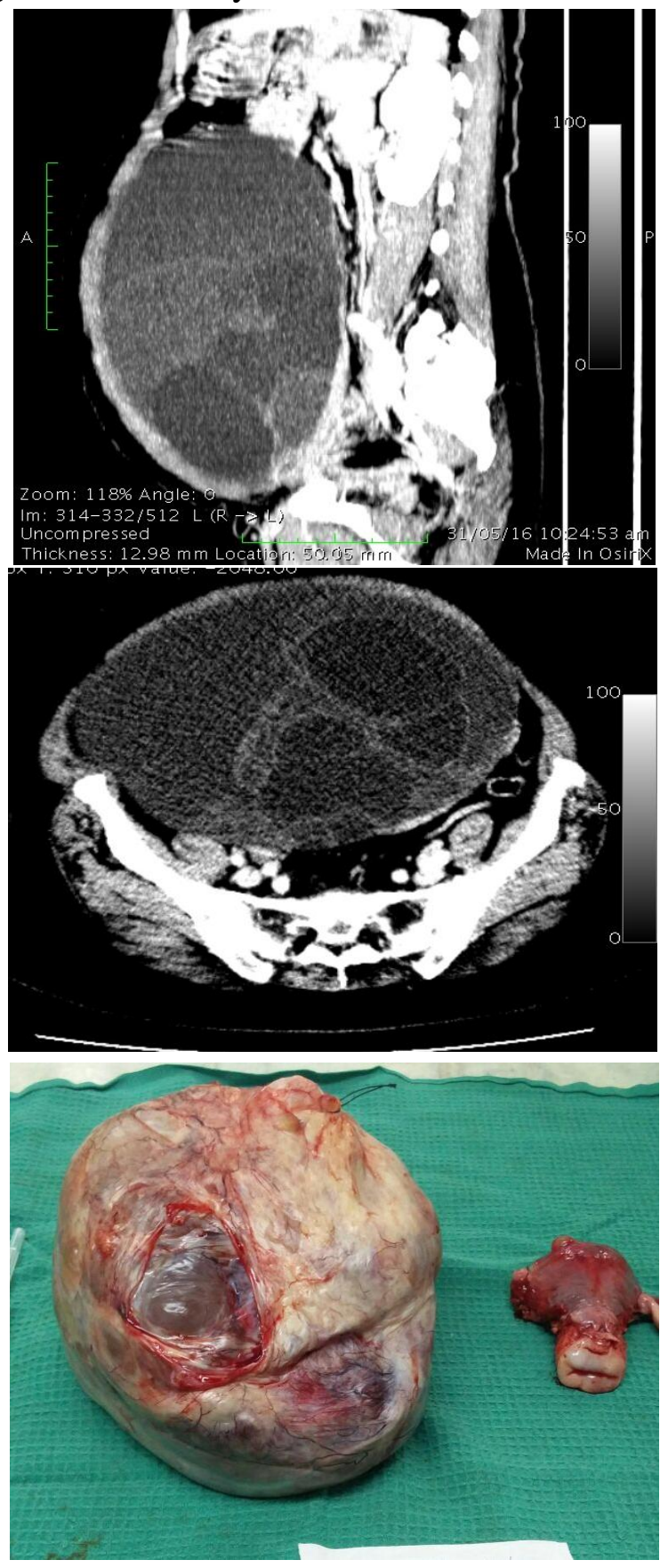

Fig-2: Serous cystadenoma

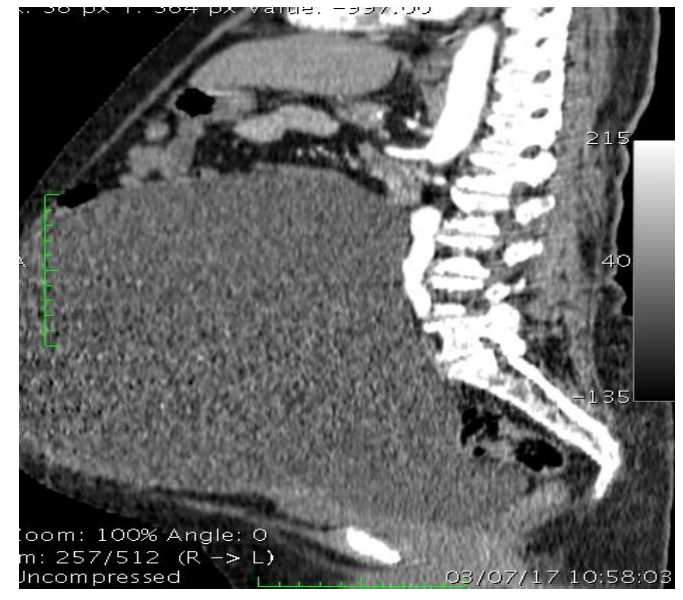

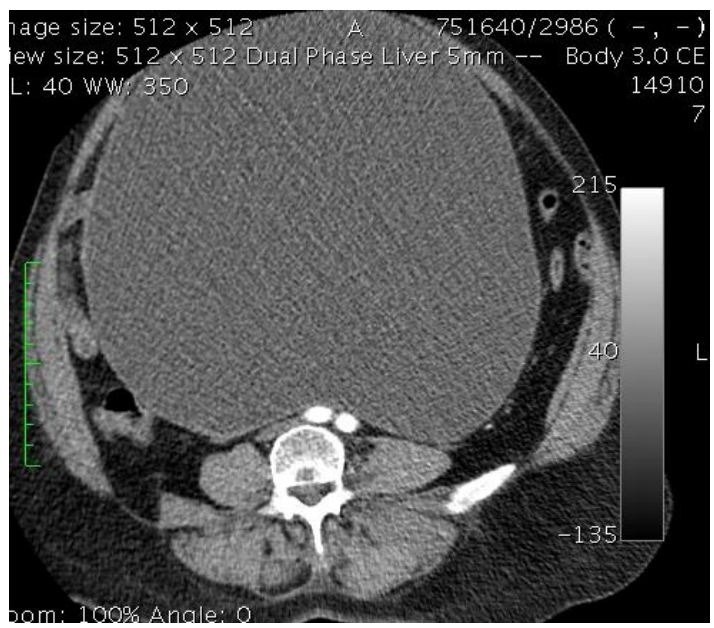

Fig-3: Gastro Intestinal Stromal tumor

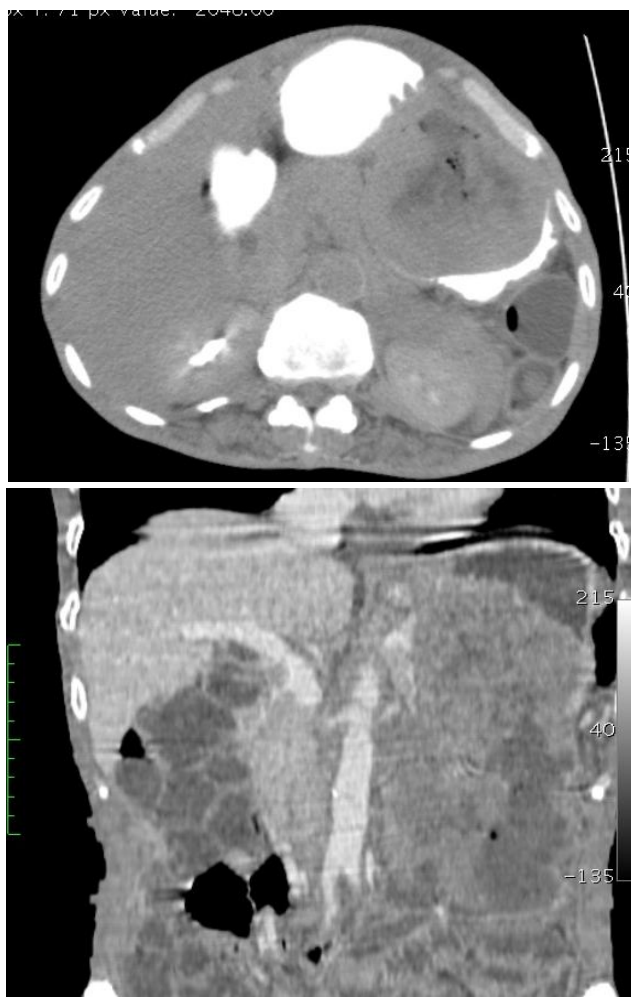

Fig-4: Ovarian Fibroma

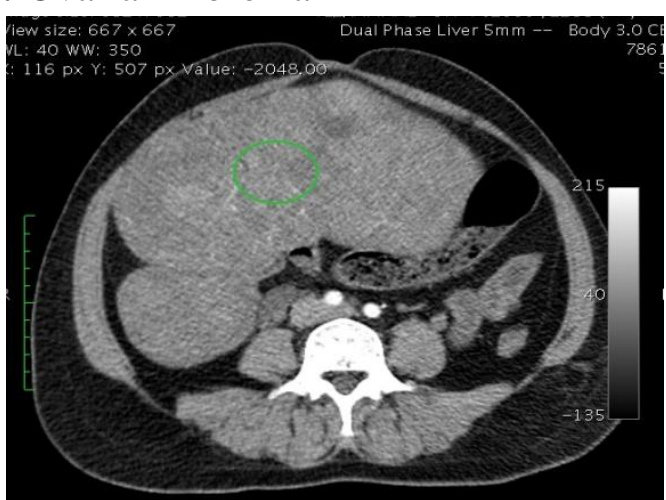




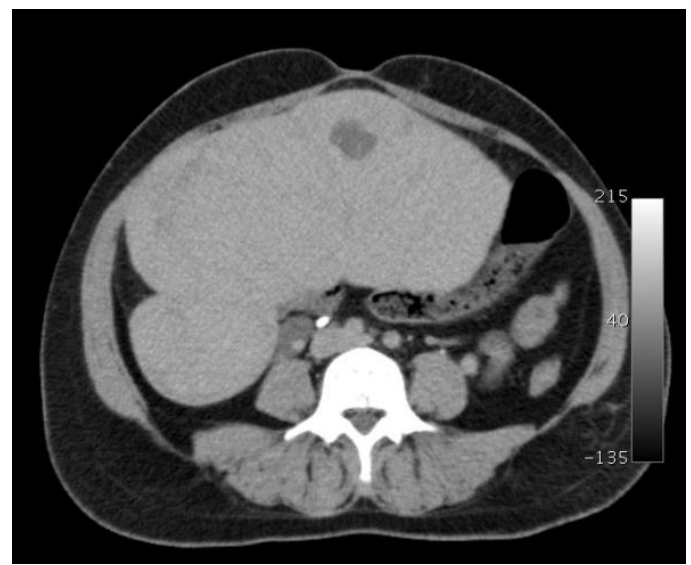

Fig-5: Pancreatic pseudocyst

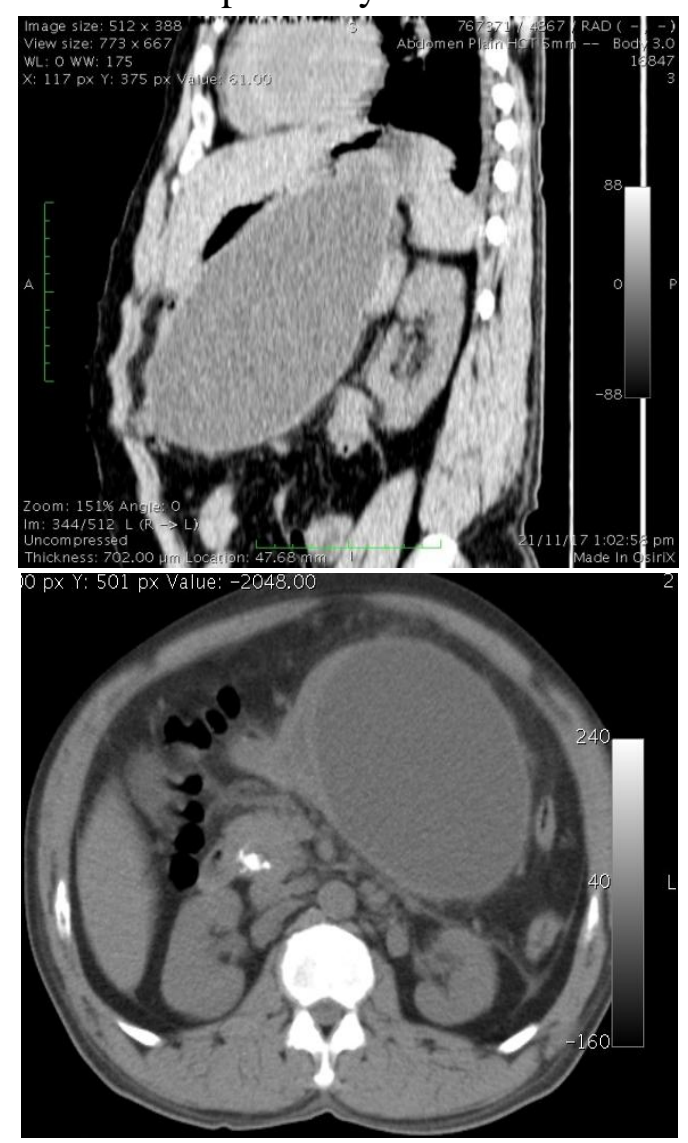

\section{Discussion}

Though ultrasound, being cost effective and easily available is the primary mode of imaging in a patient with large clinically palpable intra abdominal mass lesion, it has its own limitations with operator dependency and relative lower spatial resolution and lesser sensitivity and specificity.

Positive and negative contrast radiographs like IVU, Barium studies were less accurate in determining the tissue of origin of an intra abdominal mass lesion as compared to CT. The radiation dose received by the patient is quite high who undergoes repeated contrast radiological procedures as compared to single examination of CT. ${ }^{[1]}$

$\mathrm{CT}$ has now become the primary diagnostic modality used in oncologic radiology. The advent of Multi Slice and Multi detector CT (MSCT \& MDCT) has significantly improved the three dimensional spatial resolution and reduced the exam time resulting in high patient compliance and increases the diagnostic accuracy with multiple phases and acquisition during contrast administration $^{[2]}$. Arterial, portal and venous phase studies coupled with delayed parenchymal study have resulted in reduced need for conventional CT angiographic studies. the only limitation of contrast enhanced CT study is patients with compromised renal functions. Coronal and sagittal reformations along with the axial images assist the reporting radiologist to confidently assess the mass lesion regarding organ of origin, spread and adjacent organ involvement and also in assessing treatment follow up. Analysing the images in all orthogonal planes gives the surgeon to plan his intervention with a pre surgical road-map. ${ }^{[3]}$

MRI with its better soft tissue characterisation and direct multi planar images also has its own limitations. With patients having deranged renal parameters and those with allergic to iodine, MRI is done in lieu of CT though its cost and relatively reduced availability limits its use. Few more of its limitations include are patients with pacemakers and intra cranial aneurysmal clips where MRI is contra indicated.

PET using (18F)-Fluorodeoxy-D-glucose (FDG) is an emerging imaging technique based on the increased signal from glucose metabolism in the tissues of tumours. PET-CT has added advantage of fusing the CT images with isotope uptake images and hence detection of small primary or secondary becomes easier and it is hard to miss even subcentimeter lesions. ${ }^{[4]}$ It primarily helps in differentiating benign and malignant masse but a significant overlap between the two happen since inflammatory and infective lesions also have 
increased glucose uptake. PET using Gallium68[ga-68] DOTA-NAC has been very useful in detection of neuro endocrine tumours and metastasis with clinical finding of increased tumour markers. ${ }^{[5]}$

Of the total 178 adnexal masses in a study by Sung Il Jung, MD et al, 133 cases of patients with large abdominal mass were benign and 45 masses were malignant. However, in our study, ovarian masses were of benign and malignant nature in $50 \%$ of cases each. ${ }^{[6]}$ Recent innovations in multidetector computed tomography (MDCT), which allows thinner sections, the characterisation of adnexal mass lesions were never easy before with the advent of multi detector CT images with thinner sections and reduced timing and a good resolution of the images for staging and assessment of post-treatment follow up..$^{[7,8,9]}$

Mesenchymal tissues of the mesentery is rare to have primary tumour arising from it. Mesentery surrounding the small bowel loops form a common source of spread of tumours in the intra peritoneal space. ${ }^{[10]}$ There are commonly four ways of spread of tumour to the mesentery. Direct extension, lymphatic, hematologic and seedling metastases are the common routes. In our study, we have seen 5 cases of benign mesenteric cysts.

Haney Heneidy et al in a study predicted the pathological nature of pancreatic cystic lesions through CT examination and categorised them as benign, malignant or premalignant ${ }^{[11]}$. Our results suggested the CT features of lobulated, thin wall less than $1 \mathrm{~mm}$ as benign cystic lesions, whereas oval or round shaped complex appearing cystic lesion with the wall thickness more than $1 \mathrm{~mm}$ as probable malignant lesions. In our study, we have seen one case of pancreatic pseudo cyst.

Gastrointestinal stromal tumours (GISTs) are the common nonepithelial tumours of the gastrointestinal tract. Cajal cells of interstitium give rise to GIST. The CT characteristics of GIST are well described by Xie Hong et al. ${ }^{[12]}$ On CECT, primary GISTs are large exophytic and hyper vascular lesions. The characteristic feature of GIST are its enhancing tumour nodules. The more homogenous the lesion becomes, there is more suggestion of positive response to treatment and the tumour becomes more hypo dense too. The disappearance and reappearance of the tumour nodule represent regression and recurrence of tumour post treatment respectively. We have reported two cases of GIST in this study.

One of the commonest benign tumours of female pelvis is uterine fibroid. They form a good proportion of incidental findings and are easily detectable by USG, When the size of the fibroid becomes more increased, it adds to confusion and CT helps us in accurate delineation of the organ or origin and its benignity. Unusual appearances and characterisation have been described in degenerate fibroids ${ }^{[13]}$. We have reported two cases of Uterine fibroids in our study. Abdominal Koch's, Enlarged Gall bladder, Large hepatic and renal cysts form the rest of the cases in our study.

\section{Conclusion}

With multiplanar capabilities, increased spatial resolution, reduced scanning time $\mathrm{CT}$ is efficient in accurately predicting the organ of origin, the characteristic imaging features of large abdominal mass lesions and help in assessing the lesion to be of benign or malignant in nature ${ }^{[14]}$. Awareness of the various appearances enables prompt diagnosis and can guide treatment.

\section{Financial or other Competing interests: None.}

\section{References}

1. J C Leonidas, B L Carter, L L Leape, M L Ramesnofsky, A M Schwartz, et al. Computed tomography in diagnosis of abdominal masses in infancy and childhood. Comparison with excretory urography. Arch. Dis. Child 1978 Feb; 53(2): 120-125.

2. Ji H, McTavish JD, Mortele KJ, et al. Hepatic imaging with multidetector CT. Radiographics 2001; 21 Spec No: S71-80.

3. Smith PA, Ratner LE, Lynch FC. et al. Role of $\mathrm{CT}$ angiography in the preoperative evaluation 
for laparoscopic nephrectomy. Radiographics. 1998;18:589-601.

4. Marsden P, Sutcliffe-Goulden J. Principles and technology of PET scanning. Nucl Med Commun. 2000; 21:221-4.

5. Nunan TO, Hain SF. PET in oncology IIother tumours. Nucl Med Commun. 2000;21:229-33.

6. Sung II Jung, MD, Hee Sun Park, MD, Young Jun Kim, MD, and Hae Jeong Jeon, MD et al Multidetector Computed Tomography for the Assessment of Adnexal Mass: Is Unenhanced CT Scan Necessary? Korean J Radiol. 014 Jan-Feb; 15(1): 72-79.

7. Tsili AC, Tsampoulas C, Charisiadi A, KalefEzra J, Dousias V, Paraskevaidis E, Efremidis SC: Adnexal masses: Accuracy of detection and differentiation with multidetector computed tomography. Gynecol Oncol. 2008 Jul; 110(1):22-31.

8. Tsili AC, Tsampoulas C, Argyropoulou M, Navrozoglou I, Alamanos Y, Paraskevaidis E, Efremidis SC: Comparative evaluation of multidetector CT and MR imaging in the differentiation of adnexal masses. Eur Radiol. 2008 May; 18(5):1049-57.

9. BBB] Posabella, A., Galetti, K., Engelberger, S., Giovannacci, L., Gyr, T., \& Rosso, R. (2014). A Huge Mucinous Cystadenoma of Ovarian: A Rare Case Report and Review of the Literature. Rare Tumors, 6(2), 5225.

10. Sheila Sheth, Karen M. Horton, Melissa R. Garland, Elliot K. Fishman. Mesenteric Neoplasms: CT Appearances of Primary and Secondary Tumours and Differential Diagnosis. Radiographics. Mar 12003.

11. HaneyHeneidy, WaledYosef, Sheirin Badr et al: Cystic pancreatic lesions; CT Characterization and pathological evaluation. The Egyptian Journal of Radiology and Nuclear Medicine: Volume 48, Issue 4, December 2017, Pages 779-783

12. Xie Hong, Haesun Choi, Evelyne M. Loyer, Robert S. Benjamin, Jonathan C. Trent, Chusilp Charnsangavej: Gastrointes- tinal stromal tumor: Role of CT in diagnosis and in response evaluation and surveillance after treatment with imatinib: Radiographics. Mar 12006.

13. Wilde Sue and Scott-Barrett Sarah: Radiological appearances of uterine fibroids: Indian $\mathbf{J}$ Radiol Imaging. 2009 Aug; 19(3): 222-231.

14. Berezin, Andrew, and Steven E. Seltzer. "Differential diagnosis of huge abdominal masses visualized on CT scans." Computerized Radiology 8.2 (1984): 95-99. 